\title{
Effect of the interference with DRP1 expression on the biological characteristics of glioma stem cells
}

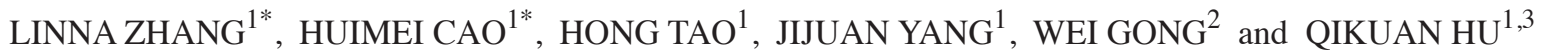 \\ ${ }^{1}$ Department of Physiology, Ningxia Medical University; ${ }^{2}$ Department of Orthopedics, Ningxia People's Hospital; \\ ${ }^{3}$ Ningxia Key Laboratory of Cerebrocranial Diseases, Basic Medical School of Ningxia Medical University, \\ Yinchuan, Ningxia Hui Autonomous Region 750004, P.R. China
}

Received October 10, 2019; Accepted January 28, 2021

DOI: $10.3892 /$ etm.2021.10128

\begin{abstract}
In the present study, a model of glioma stem cells (GSCs) was established and combined with molecular targeting drugs in order to observe its inhibitory effect on the proliferation and biological characteristics of GSCs, with the aim of providing a potential target for the treatment of glioma. On the basis of a relatively classical induction strategy with neuron induction medium, a large number of GSC-like cells in good condition and globular growth were amplified in vitro, which had the potential to differentiate into neurons, oligodendrocytes and astrocytes/glioma cells. It was observed that the interference with dynamin-related protein 1 expression using Mdivi-1, a mitochondrial mitotic inhibitor, at the optimal concentration, decreased the expression level of stem cell-associated genes, inhibited proliferation and promoted apoptosis in GSCs. The present study provided an experimental basis for a novel strategy of cancer treatment with tumor stem cells as the target.
\end{abstract}

\section{Introduction}

Glioma is an invasive malignant tumor of the central nervous system. Surgery is most commonly used for treatment, which aims to decrease the tumor volume and the number of tumor cells, relieve symptoms, prolong life and create opportunities for other treatments (1). However, residual tumor in the primary site after general surgery will still exist, which can generate progeny cells that are sufficient to sustain tumor growth, overcome general cytotoxic therapies, and eventually result in tumor recurrence (2). The continuous malignant growth of tumors is the key cause of recurrence. These types of cells

Correspondence to: Professor Qikuan $\mathrm{Hu}$, Department of Physiology, Ningxia Medical University, 1160 Shengli Street, Yinchuan, Ningxia Hui Autonomous Region 750004, P.R. China E-mail: huqikuan@163.com

*Contributed equally

Key words: glioma stem cells, mitochondrial fission, DRP1 are usually referred to as tumor-propagating cells, stem cells or persisters, and they are present in numerous high-grade malignancies, including glioblastoma (3). Only by overcoming the threat of recurrence can glioma be cured. In different types of tumor, there are few cells with self-renewal, unlimited proliferation and multiple differentiation potential, which exhibit the basic characteristics of stem cells and are known as cancer stem cells (CSCs) (4). Glioma stem cells (GSCs) have been successfully isolated from the brain glioma tissue in an in-depth study of malignant glioma (5). It has been hypothesized that there is an association between glioma recurrence and GSCs. For example, decreased asymmetry in normal stem cells has been associated with neoplastic transformation, while therapies that increase the rate of asymmetric division result in decreased numbers of resistant GSCs (6). In addition, the frequency of asymmetric division of CSCs is negatively correlated with their proliferative capacity $(7,8)$. GSCs exhibit the potential of multidirectional differentiation, and can differentiate into neurons, oligodendrocytes and astrocytes (9). At present, it is generally believed that the way of differentiation of GSCs is very similar to that of neural stem cells (NSCs) (10). In addition, a number of studies have also demonstrated that GSCs can be isolated and proliferate in vitro $(11,12)$. It has also been indicated that NSCs can proliferate via symmetrical or asymmetric division (13). On the contrary, it was believed that the main mode of proliferation of GSCs was symmetrical and not asymmetric cell division (14). However, when GSCs were inoculated into the subcutaneous layer of immunodeficient mice, it was observed that they were differentiated into glioblasts in an asymmetrical manner (15). Numerous features of NSCs have also been observed in GSCs, including common surface markers, such as CD133 and Nestin $(16,17)$. Although there is an association between GSCs and NSCs, it is still essential to determine whether the induction medium of NSCs can also induce GSCs, and whether additional stem cell-associated genes in NSCs are also expressed in GSCs. Based on the aforementioned information, the present study selected a type of relatively mature culture medium $(18,19)$ of NSCs to induce GSCs in order to achieve an ideal induction effect in vitro. Therefore, the aim of the present study was to establish a glioma cell model in vitro, and further investigate the pathogenesis, antitumor drug sensitivity and molecular biological characteristics. The study of GSCs can provide 
a new direction to unravel the origin and the mechanism of development of malignant glioma, and discover novel treatments for these diseases.

Mitochondria, one of the most important organelles of the cell, are involved in a dynamic balance process of fission and fusion, which is important for maintaining the normal morphology, distribution and function of mitochondria (20) This process determines the morphology, quantity, function and spatial distribution of mitochondria, thereby affecting biological processes, such as the production of ATP, the phagocytosis of mitochondria, apoptosis and calcium homeostasis (21). Mitochondria are associated with a number of clinical diseases, such as cancer, cardiovascular diseases, neurodegenerative diseases and pulmonary hypertension (22-24). The occurrence of these diseases is often accompanied by mitochondrial dysfunction and/or structural disorders. For example, it has been demonstrated that cancer cells usually exhibit mitochondrial fragmentation, indicating that the excessive division, contraction or decreased fusion of mitochondria is associated with the occurrence of cancer; however the specific underlying mechanism remains unclear (25). The alterations in the expression levels of fusion- and fission-associated proteins directly affect the process of mitochondrial fusion and fission, which indicates that defects in proteins involved in the mitochondrial dynamics that regulate mitochondrial fusion and fission can affect cellular differentiation, proliferation, cellular reprogramming and aging (26).

Dynamin-related protein 1 (DRP1), which has GTPase activity, is an essential protein in the process of mitochondrial division. As a major protein, it is activated and located on the mitochondrial membrane via non-GTP receptor proteins, such as mitochondrial fission 1 protein (Fis1), mitofusins (Mfns) and mitochondrial elongation factor, forming a circular structure, which contracts and divides the mitochondria. The fission process may involve DRP1, Mfns and the microregions of pro-apoptotic proteins (27). Ji et al (28) suggested that actin induced the recruitment of DRP1 to the mitochondria to promote mitochondrial fission, and also demonstrated that actin filaments were localized close to DRP1 on the mitochondrial membrane to increase the probability of mitochondrial division before DRP1 is recruited to the mitochondria. Mitochondrial division is a multistep process, which is initiated by the recruitment of Fis1 on the outer mitochondrial membrane, where DRP1 is transposed to and enriched in potential mitotic sites (29). Several DRP1 molecules form ring structures around the mitochondria, alter the distance or angle between molecules by the hydrolysis of GTP, and gradually compress the mitochondria until they divide, resulting in two independent mitochondria (30). Similar to mitochondrial fission, Mfn1 and Mfn2, which are two Mfn isoforms with a central action in tethering and fusion, are localized in the outer mitochondrial membrane in mammals (27). However, the GTPase and tethering actions of Mfn1 are more pronounced than that those of Mfn2 (31). After mitochondrial division, DRP1 returns to the cytoplasm, and the cycle is repeated. By using Mdivi-1, which is a selective and transmembrane mitochondrial mitotic inhibitor, to interfere with the kinetic equilibrium of mitochondria, the role of DRP1 can be more easily examined (32). However, to the best of our knowledge, the interaction mechanism between Mdivi-1 and DRP1 has rarely been reported. Therefore, investigating the effects on biological characteristics of GSCs by interference with DRP1 expression is a current field of study. Mdivi-1, bound to an allosteric site of DRP1 impeding its self-assembly and GTP hydrolysis, has been indicated to prevent mitochondria fission resulting in inter-connected, net-like mitochondria (33). It has been indicated that Mdivi-1 restored mitochondrial network organization and energy production (34). However, the effect of Mdivi-1 on the induction and differentiation of GSCs has not yet been investigated. Therefore, the present study aimed to establish a drug-targeting platform with GSCs as a cell model and DRP1 as a mitochondrial target. Mdivi-1, a molecular targeting drug, may further inhibit the general stem cell characteristics of GSCs, thereby increasing the sensitivity of GSCs to drugs. This method is likely to provide an important target for the treatment of glioma. At the same time, it provides a certain experimental basis for a novel strategy of cancer treatment with tumor stem cells as a target. Metabolic research has been indicated to be an important tool in the identification of novel treatments against cancer (35). However, the role of mitochondrial metabolism in tumor development remains unclear. If the current understanding of mitochondrial dynamic regulation and its intrinsic significance to the maintenance and proliferation of GSCs is improved, it may become a powerful tool for tumor treatment.

\section{Materials and methods}

Experimental group. When Mdivi-1 (cat no. ab144589; Abcam) interfered with GSCs, they were divided into 7 groups according to different treatment conditions at $37^{\circ} \mathrm{C}$ in humidified air with $5 \% \mathrm{CO}_{2}$. The 7 groups were as follows: M1-5d (continuous treatment for 5 days with $1 \mu \mathrm{M}$ Mdivi-1), M5-2d (continuous treatment for 2 days with $5 \mu \mathrm{M}$ Mdivi-1), M5-5d (continuous treatment for 5 days with $5 \mu \mathrm{M}$ Mdivi-1), M5-7d (continuous treatment for 7 days with $5 \mu \mathrm{M}$ Mdivi-1), M10-2d (continuous treatment for 2 days with $10 \mu \mathrm{M}$ Mdivi-1), M10-5d (continuous treatment for 5 days with $10 \mu \mathrm{M}$ Mdivi-1) and M10-7d (continuous treatment for 7 days with $10 \mu \mathrm{M}$ Mdivi-1). They were compared with normal control group (untreated GSCs).

Cell lines and culture. The U87 cell line was purchased from The Cell Bank of Type Culture Collection of Chinese Academy of Sciences, and it is a glioblastoma cell line but whose origin is unknown. It was identified by short tandem repeat profiling by Procell Life Science \& Technology Co., Ltd. A maximum number of 5 cell passages were used before analysis. The cells were routinely cultured in DMEM complete medium (Gibco; Thermo Fisher Scientific, Inc.) with 10\% FBS (Gibco; Thermo Fisher Scientific, Inc.) and 1\% Penicillin/Streptomycin (Thermo Fisher Scientific, Inc.). The N3 medium, included the minimum essential medium/F12 basic medium (Gibco; Thermo Fisher Scientific, Inc.), $25 \mu \mathrm{g} / \mathrm{ml}$ insulin (cat. no I6040; Beijing Biotopped Science \& Technology Co., Ltd.), $50 \mu \mathrm{g} / \mathrm{ml}$ transferrin (cat. no T6010; Beijing Biotopped Science \& Technology Co., Ltd.), 30 nM sodium selenite (cat. no 214485; Sigma Aldrich; Merck KGaA), 20 nM progesterone (cat. no IP0400; Beijing Solarbio Science \& Technology Co., Ltd.), $100 \mathrm{nM}$ putrescine 
(cat. no D6140; Beijing Biotopped Science \& Technology Co., Ltd.) and 1\% penicillin/streptomycin (Thermo Fisher Scientific, Inc.). Unlike U87, for the 6 days of induction and maintenance of GSCs the cells were cultured in N3 medium with $10 \mathrm{ng} / \mathrm{ml}$ epidermal growth factor and $10 \mathrm{ng} / \mathrm{ml}$ basic fibroblast growth factor (both from ProteinTech Group, Inc.), which is also named N3EF. All cells were cultured at $37^{\circ} \mathrm{C}$ in an atmosphere containing $5 \% \mathrm{CO}_{2}$.

Tumorsphere formation assay. U87 cells were cultured in DMEM complete medium in six-well culture plates with a density of $\sim 1 \times 10^{4} / \mathrm{cm}^{2}$. When the cell density was $>60 \%$ the medium was changed to N3EF that is suitable for the growth of globular cells. After 2 days small clone-like cells appeared and the medium was refreshed every other day. On the 6 th day, the globular cells were disrupted gently with a pipette tip to prepare single cells, and the cells were collected after centrifugation at $200 \mathrm{x}$ g for $5 \mathrm{~min}$ at room temperature.

Calculation of tumor sphere proliferation efficiency using the Cell Counting Kit-8 (CCK-8) assay. Following preparation of cell suspension as aforementioned, the cells were seeded in a 96-well culture plate and cultured in a $37^{\circ} \mathrm{C}$ incubator with $5 \% \mathrm{CO}_{2}$. The cells were continuously observed for 7 days. Tumorspheres were formed and proliferation was measured using CCK-8 assay (cat. no BB4202, BestBio Science). A total of $10 \mu \mathrm{l}$ CCK- 8 reagent was added to $100 \mu$ l culture medium per well at $37^{\circ} \mathrm{C}$ for $3 \mathrm{~h}$. The absorbance at $450 \mathrm{~nm}$ was measured every day for 7 days. The average absorbance values of the experimental group and the control group were recorded, and proliferation efficiency was calculated according to the following equation: Cell proliferation $=[(\mathrm{OD}-\mathrm{blank}$ OD)/(control cell OD-blank OD)].

Western blot analysis. U87 cells or GSCs were washed twice in cold PBS, and subsequently lysed in cold lysis buffer from the Whole Cell Lysis assay (cat. no KGP250, Nanjing KeyGen Biotech Co., Ltd.) according to the manufacturer's protocol. The lysate was centrifuged at $10,000 \mathrm{x}$ g for $20 \mathrm{~min}$ at $4^{\circ} \mathrm{C}$. The supernatant was collected, and the protein concentration was determined using the BCA Protein Quantitation assay (cat. no KGPBCA; Nanjing KeyGen Biotech Co., Ltd.), according to the manufacturer's protocol. A total of $30 \mu \mathrm{g}$ protein/lane were separated by SDS-PAGE (8-10\% gel), and then transferred to PVDF membranes. Subsequently, the membranes were blocked with 5\% skimmed milk (cat. no. 232100; Difco; BD Biosciences) for $90 \mathrm{~min}$ at room temperature and incubated with primary antibodies at $4^{\circ} \mathrm{C}$ overnight. The antibodies were as follows: CD133 (1:300; cat. no. bs-0395R; BIOSS), Krueppel-like factor 4 (Klf4; 1:600; cat. no. 11880-1-AP), Nanog (1:600; cat. no. 14295-1-AP), SOX2 (1:600; cat. no. 20118-1-AP), c-Myc (1:600; cat. no. 10828-1-AP), OCT4 (1:1,000; cat. no. 60242-1-Ig), Nestin (1:600; cat.no. 19483-1-AP), DRP1 (1:1,000; cat. no. 12957-1-AP), Mfn1 (1:1,000; cat. no. 13798-1-AP), Fis1 (1:1,000; cat. no. 0956-1-AP), $\beta$-actin (1:5,000; cat. no. 66009-1-Ig), GAPDH (1:5,000; cat. no. 60004-1-Ig) (all from ProteinTech Group, Inc.), Bax (1:1,000; cat.no. 2772), Bcl-2 (1:1,000; cat. no. 3498) and cleaved caspase-3 (1:1,000; cat. no. 14220) (all from Cell Signaling Technology, Inc.). The membranes were washed with PBST (PBS and 0.1\% Tween-20) and incubated with IRDye ${ }^{\circledR} 680 \mathrm{RD}$
Table I. Primer sequences.

\begin{tabular}{ll} 
Primer name & \multicolumn{1}{c}{ Primer sequences } \\
\hline CD133 & F: 5'-TTCTATGCTGTGTCCTGGGGC-3' \\
& R: 5'-TTGTTGGTGCAAGCTCTTCAAGGT-3' \\
oCT4 & F: 5'-CAGCGACTATGCACAACGAGAGG-3' \\
& R: 5'-CCAGAGTGGTGACGGAGACAGG-3' \\
Nanog & F: 5'-TGCATGCAGTTCCAGCCAAA-3' \\
& R: 5'-ACACGTCTTCAGGTTGCATGT-3' \\
SOX2 & F: 5'-GACCAGCTCGCAGACCTACA-3' \\
& R: 5'-TCGGACTTGACCACCGAAC-3' \\
C-MYC & F: 5'-CTCAGAGAAGCTGGCCTCCTACC-3' \\
& R: 5'-GCGAGCTGCTGTCGTTGAGAG-3' \\
Nestin & F: 5'-TGCCTAGTTCTTCTGCTATCCT-3' \\
& R: 5'-GGGAAGCTCTGATCCTCTTTC-3' \\
Klf4 & F: 5'-CGTCGGTCATCAGCGTCAGC-3' \\
& R: 5'-CCGCCTCCTGCTTGATCTTGG-3' \\
F-actin & F: 5'-CCACACCCGCCACCAGTTCG-3' \\
& R: 5'-TACAGCCCGGGGAGCATCGT-3' \\
GAPDH & F: 5'-CAAGGTCATCCATGACAACTTTG-3' \\
& R: 5'-GTCCACCACCCTGTTGCTGTAG-3'
\end{tabular}

F, forward; R, reverse.

Goat anti-Rabbit IgG (1:5,000; cat. no. 926-68071, LI-COR Biosciences) and IRDye ${ }^{\circledR}$ 680RD Goat anti-Mouse IgG (1:5,000; cat. no. 925-68070, LI-COR Biosciences) secondary antibodies for $1 \mathrm{~h}$ at room temperature. All antibodies were diluted in 5\% skimmed milk. Finally, the PVDF membranes (Millipore, lnc) were quantified using Odyssey Infrared Imaging System, Image Studio version 4.0 software (LI-COR Biosciences).

Reverse transcription-quantitative PCR (RT-qPCR). RNA was extracted from cells (both U87 and GSCs) using the AxyPrep Multisource RNA Miniprep Kit (Axygen; Corning,Inc.). cDNA was performed with the GoScript ${ }^{\mathrm{TM}}$ Reverse Transcriptase System kit (cat. no. A5001, Promega Corporation), according to the manufacturer's instructions-PCR amplification was performed using Tip Green qPCR SuperMix (TransGen Biotech Co., Ltd.) in a $20 \mu \mathrm{l}$ reaction containing Tip Green qPCR SuperMix buffer, primers and diluted cDNA. The primer sequences are listed in Table I. The PCR cycling conditions were as follows: $94^{\circ} \mathrm{C}$ for $30 \mathrm{~min}$, followed by 40 cycles of $94^{\circ} \mathrm{C}$ for $30 \mathrm{sec}, 50-60^{\circ} \mathrm{C}$ for $30 \mathrm{sec}$ and $72^{\circ} \mathrm{C}$ for $1 \mathrm{~min} / \mathrm{kb}$. All relative expression values were normalized to GAPDH levels using the $2^{-\Delta \Delta \mathrm{Cq}}$ method (36).

Cell immunofluorescence staining. GSCs were fixed using $4 \%$ polyformaldehyde at room temperature for $15 \mathrm{~min}$. After washing 3 times with PBS, the cells were incubated with $0.5 \%$ Triton X-100 in PBS for $20 \mathrm{~min}$ at room temperature. Subsequently, the cells were incubated with $10 \%$ goat serum (OriGene Technologies, Inc.) for $30 \mathrm{~min}$ at room temperature. The liquid was discarded, and the cells were immersed in the primary antibodies: Rabbit anti-glial fibrillary acidic protein (GFAP; 1:200; cat. no. 16825-1-AP; ProteinTech Group, 

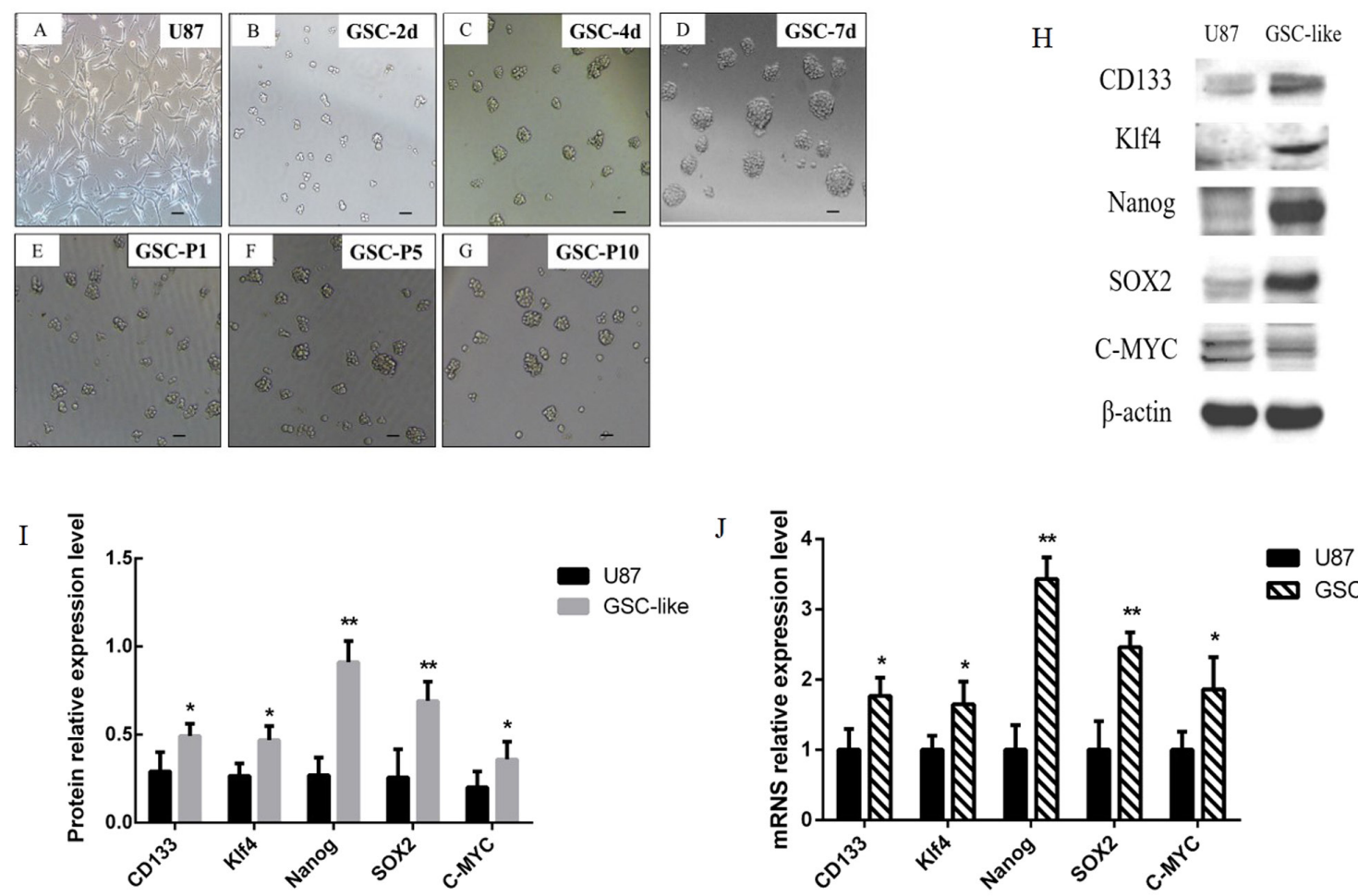

Figure 1. Morphological alterations of GSC-like cells were observed on different days, and expression of tumor stem cell-associated genes was detected by western blotting and RT-qPCR. (A) Glioma cells. (B) GSC-2 days. (C) GSC-3 days. (D) GSC-7 days. (E-G) GSC-P1, P5 and P10. Scale bars, $50 \mu \mathrm{m}$. (H) The expression level of CD133 (130 kDa), c-Myc (57-70 kDa), Klf4 (65 kDa), SOX2 (35 kDa), Nanog (42 kDa) and $\beta$-actin $(42 \mathrm{kDa})$ was examined by western blotting. (I) Grayscale analysis results of western blotting. (J) CD133, c-Myc, K1f4, SOX2 and Nanog expression at the mRNA level was analyzed by RT-qPCR and normalized to $\beta$-actin. ${ }^{*} \mathrm{P}<0.05$ and ${ }^{* *} \mathrm{P}<0.01$ vs. U87 as control group. GSC, glioma stem cells; Klf4, Krueppel-like factor 4; P1, passage 1; P5, passage 5; P10, passage 10; d, day; RT-qPCR, reverse transcription-quantitative PCR.

Inc.), mouse anti-O4 (1:200; cat. no. O7139; MilliporeSigma) and mouse anti-tubulin beta-3 chain (Tuj1; 1:250; cat. no. 480011; Invitrogen; Thermo Fisher Scientific, Inc.) at $4^{\circ} \mathrm{C}$ overnight. Following incubation of the cells for $30 \mathrm{~min}$ at room temperature in the dark with Alexa Fluor ${ }^{\mathrm{TM}} 555$ goat anti-mouse $\lg$ (1:500; cat. no. A21422; Invitrogen; Thermo Fisher Scientific, Inc.) or Alexa Flour ${ }^{\mathrm{TM}} 488$ goat anti-rabbit $\lg G$ (1:500; cat. no. A11008; Invitrogen; Thermo Fisher Scientific, Inc.), the nuclei were stained using DAPI at room temperature for $10 \mathrm{~min}$. The stained cells were observed under a confocal microscope at $\mathrm{x} 20$ magnification.

Statistical analysis. Each experiment was repeated three times, and all data are presented as the mean \pm SD. Statistical analyses were performed using SPSS v22.0 software (IBM Corp.). Comparisons between two groups were performed using independent samples Student's t-test, and for comparisons among multiple groups, one-way ANOVA followed by Bonferroni's post hoc test was used. $\mathrm{P}<0.05$ was considered to indicate a statistically significant difference. Quantification analysis of the GSC sphere numbers using ImageJ v1.47 software (National Institutes of Health). Cell sphere diameter $\geq 50 \mu \mathrm{m}$ was considered to indicate a GSC sphere.

\section{Results}

Identification of glioma-like stem cells in vitro. Following culture of U87 cells (Fig. 1A) at the first day. Morphological alterations were observed in certain cells on the 2nd day
(Fig. 1B) with N3EF medium. It was revealed that the majority of cells grew in spherical suspension, and the volume of the sphere increased markedly (Fig. 1C). On the 7th day, it was revealed that the majority of cells were larger (diameter $\geq 50 \mu \mathrm{m}$; Fig. 1D). After passaging, the cells were in a good condition the following days, which was considered as the morphological characteristics of the cells being clear and cell growth being high (Fig. 1E-G).

RT-qPCR data demonstrated that the CD133, C-MYC andKIf4mRNA expression levels were significantly increased in GSC-like cells $(\mathrm{P}<0.05$; Fig. $1 \mathrm{~J})$. Notably, the changes in mRNA expression levels of SOX2 and Nanog were the most significant $(\mathrm{P}<0.01$; Fig. 1J). It indicated that the cells exhibited characteristics of tumor stem cells. The CD133, C-MYC and Klf4 protein expression levels detected by western blot analysis were markedly increased in GSC-like cells compared with the control $(\mathrm{P}<0.05$; Fig. $1 \mathrm{H}$ and I). Consistent with the trend of RT-qPCR results, the expression of SOX2 and Nanog increased more significantly at the protein level $(\mathrm{P}<0.01$; Fig. $1 \mathrm{H}$ and I). These data indicated that the GSC-like cells exhibited characteristics of stem cells in vitro at both the mRNA and protein level.

Detection of the differentiation potential of GSC-like cells. GSCs have the potential of multidirectional differentiation $(37,38)$. Neuron marker protein Tuj1 (Fig. 2A), oligodendrocyte marker protein O4 (Fig. 2B) and astrocyte marker protein GFAP (Fig. 2C) were observed to be expressed after induction of GSC-like cells for 30, 25 and 7 days 



$\mathrm{B}$
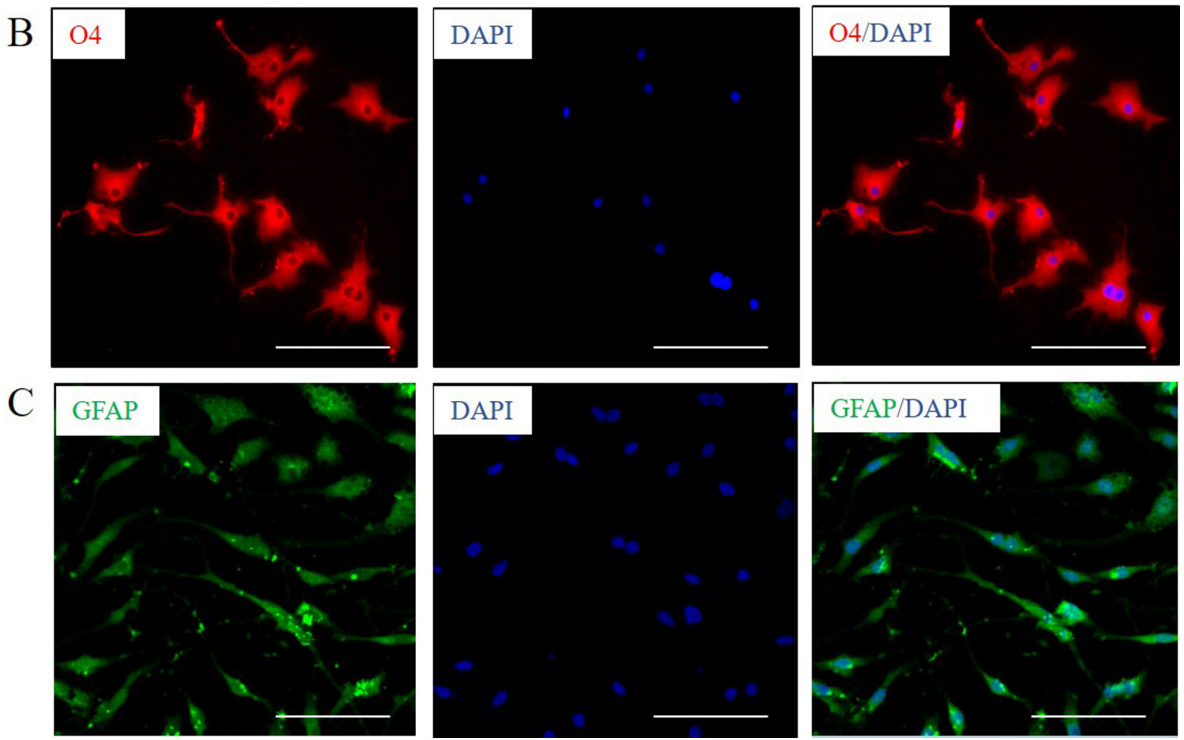

Figure 2. Detection of the differentiation potential of glioma stem cells by immunofluorescence. (A) Neuronal marker protein Tuj1 (red). (B) Oligodendrocyte marker protein O4 (red). (C) Astrocyte cell marker protein GFAP (green). Scale bars, $20 \mu$ m. Tuj1, tubulin beta-3 chain; GFAP, glial fibrillary acidic protein.

respectively, by immunofluorescence. The results revealed that GSC-like cells exhibited a certain multidirectional differentiation potential. It can be determined that the GSC-like cells have the characteristics of stem cells, and the present induction system can be used for the in vitro culture of GSCs.

Protein expression of mitochondrial-associated genes in GSCs. The expression levels of the mitotic protein DRP1 and the fusion protein Mfn1 in the control cell group and GSC cell group were analyzed. The results revealed that expression of DRP1 was significantly upregulated in the GSC group compared with the control group $(\mathrm{P}<0.01$; Fig. 3). However, there was no significant difference in the expression of Mfn1 (P>0.01; Fig. 3). These results demonstrated that there was abnormal division of the mitochondria in GSCs.

Intervention in GSCs with Mdivi-1 alters the expression of DRPI and Fisl at different concentrations and time points. Mdivi-1 was used to intervene in the mitochondrial division of GSCs, and firstly the optimal intervention concentration of Mdivi-1 was determined. A total of five concentrations were selected according to the pharmacological properties of Mdivi-1 (39); 1, 5, 10, 20 and $50 \mu \mathrm{M}$. After intervention with different concentrations, it was preliminarily observed that the cells could not survive at a concentration of 20 and $50 \mu \mathrm{M}$, by observing the morphology and growth status of the cells (Fig. 4). In order to identify the most suitable Mdivi-1 concentration, the present study further performed a screening of the Mdivi-1 intervention concentrations and time points by detecting the protein expression alterations of DRP1 and Fis. 1. Compared with the normal control group (untreated GSCs), the expression levels of DRP1 and Fis1 were more importantly decreased in the M5-5d group $(\mathrm{P}<0.01$; Fig. 5). It was therefore established that subsequent experiments could be performed with the M5-5d dose.

Effect on stem cell characteristics of GSCs with intervention of $5 \mu M$ Mdivi-1 for 5 days. The mRNA expression levels of CD133, c-Myc, Klf4, SOX2, Nestin, Nanog and OCT4 in the control GSC group and the GSC group treated with a continuous intervention of $5 \mu \mathrm{M}$ Mdivi- 1 for 5 days were analyzed by RT-qPCR assay. The results revealed that the mRNA expression levels of these genes decreased significantly after Mdivi-1 intervention (all $\mathrm{P}<0.001)$. The CD133 ( $\mathrm{P}<0.01)$, c-Myc $(\mathrm{P}<0.001)$, Klf4 $(\mathrm{P}<0.01)$, SOX2 $(\mathrm{P}<0.001)$, Nestin $(\mathrm{P}<0.05)$, Nanog $(\mathrm{P}<0.01)$ and OCT4 $(\mathrm{P}<0.05)$ protein expression levels detected via western blot analysis were markedly decreased in Mdivi-1 treated cells compared with the GSC group (Fig. 6). These results revealed that the expression level of the majority of stem cell-associated genes in GSCs was notably reduced following continuous treatment of the GSCs with $5 \mu \mathrm{M}$ Mdivi-1 for 5 days.

Effects on the proliferation and apoptosis of GSCs after treatment with $5 \mu \mathrm{M}$ Mdivi-1 for 5 days. After $5 \mu \mathrm{M}$ Mdivi-1 continuous intervention for 5 days(M5-5), the number of 
A

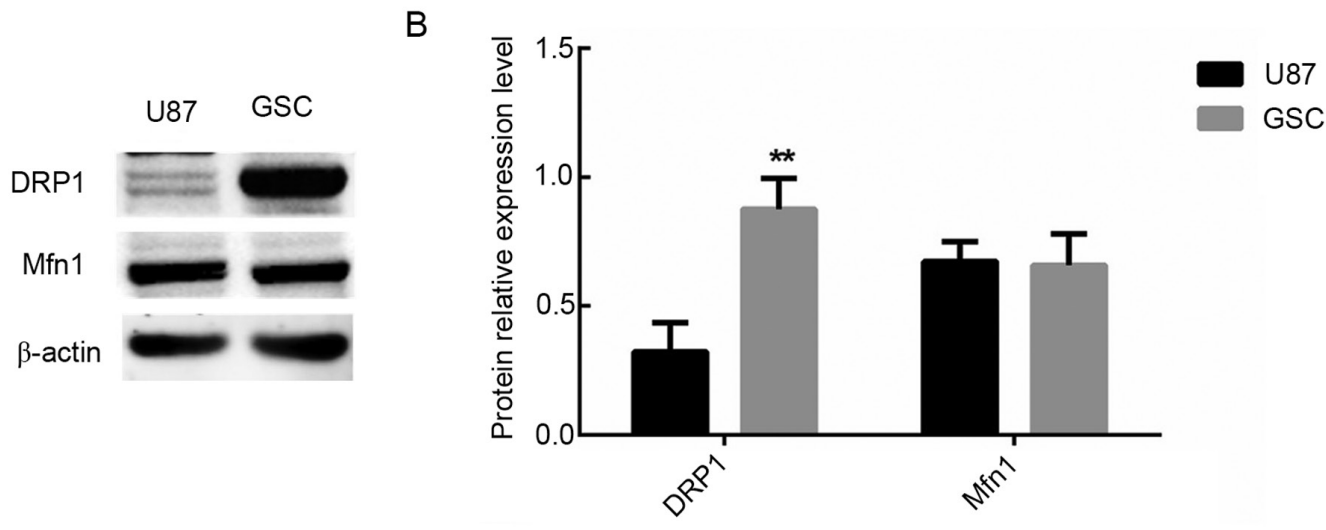

Figure 3. Protein expression levels of mitochondria-associated genes in GSCs. (A and B) DRP1 (78-82 kDa) and Mfn1 (82 kDa) were analyzed via western blotting, and $\beta$-actin (42 kDa) was used as an internal control. ${ }^{* *} \mathrm{P}<0.01$ vs. U87 as control group. GSC, glioma stem cells; DRP1, dynamin-related protein 1; Mfn1, mitofusin-1.

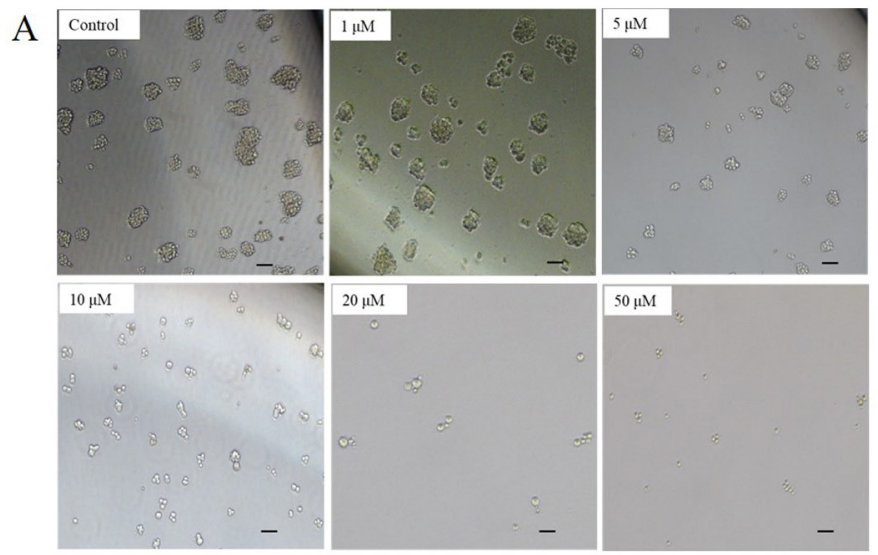

$\mathrm{B}$

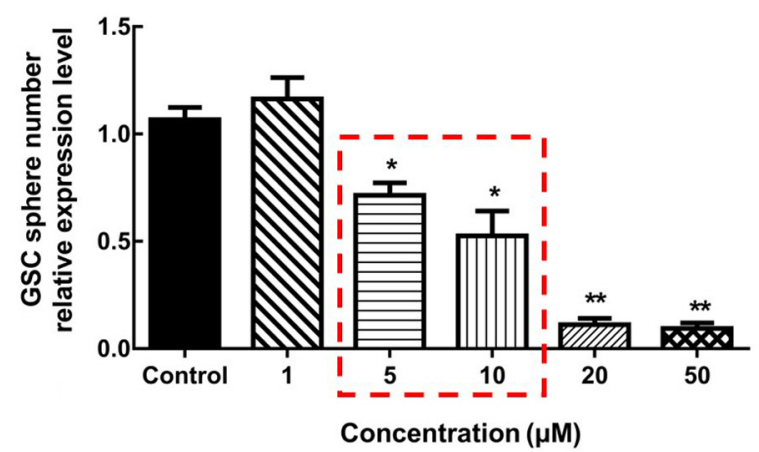

Figure 4. GSC number alterations after Mdivi-1 intervention with different concentrations. (A) Morphology of cells observed under the optical microscope. Scale bars, $50 \mu \mathrm{m}$. (B) Quantification analysis of the GSC sphere numbers using ImageJ software. GSC, glioma stem cells. " $\mathrm{P}<0.05$ and ${ }^{* *} \mathrm{P}<0.01 \mathrm{vs}$. untreated GSCs as control group.

A

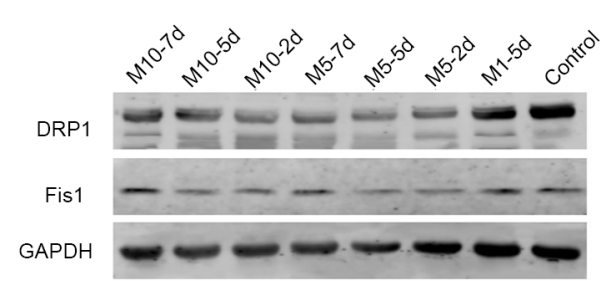

B

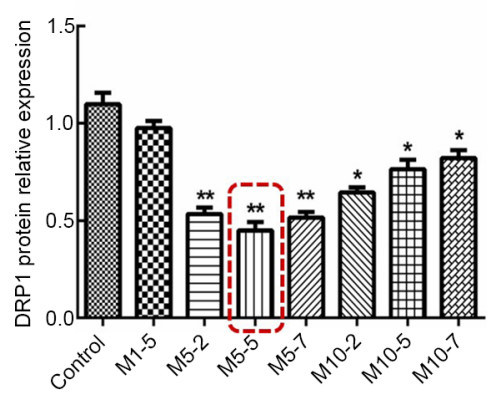

C

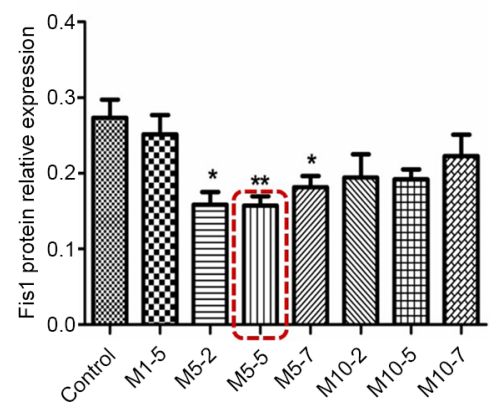

Figure 5. Detection of DRP1 and Fis1 expression after intervention with different Mdivi-1 concentrations and durations via western blotting. (A-C) The protein expression levels of DRP1 (78-82 kDa) and Fis1 (17 kDa) were detected after intervention of $1 \mu \mathrm{M}$ Mdivi-1 for 5 days, $5 \mu \mathrm{M}$ Mdivi-1 for 2 days, $5 \mu \mathrm{M}$ Mdivi- 1 for 5 days, $5 \mu \mathrm{M}$ Mdivi-1 for 7 days, $10 \mu \mathrm{M}$ Mdivi-1 for 2 days, $10 \mu \mathrm{M}$ Mdivi-1 for 5 days and $10 \mu \mathrm{M}$ Mdivi-1 for 7 days. ${ }^{*} \mathrm{P}<0.05$ and ${ }^{* *} \mathrm{P}<0.01$ vs. untreated GSCs as control group. DRP1, dynamin-related protein 1; Fis1, mitochondrial fission 1 protein; M, Mdivi-1; d, day.

globular GSCs was reduced compared to normal untreated GSCs cultured continuously for 5 days (data not shown).And the treated cells were selected for subsequent experiments (Fig. 7A). The results of the CCK-8 assay revealed that the proliferative ability of GSCs was markedly inhibited following 1 week of Mdivi-1 intervention $(\mathrm{P}<0.001$; Fig. 7B).
The expression levels of apoptosis-associated proteins in the control group (GSC group) and the experimental group (GSCs treated with $5 \mu \mathrm{M}$ Mdivi-1 for 5 days) was analyzed via western blotting. The results revealed that compared with the GSC group, the expression levels of the apoptosis promoting factors Bax and cleaved caspase-3 were significantly increased 
A

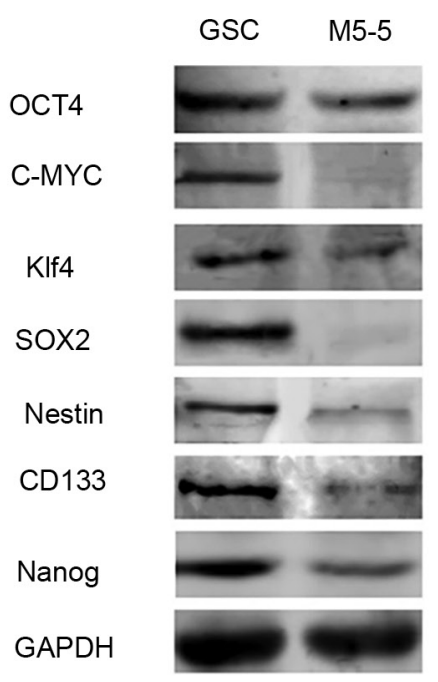

B

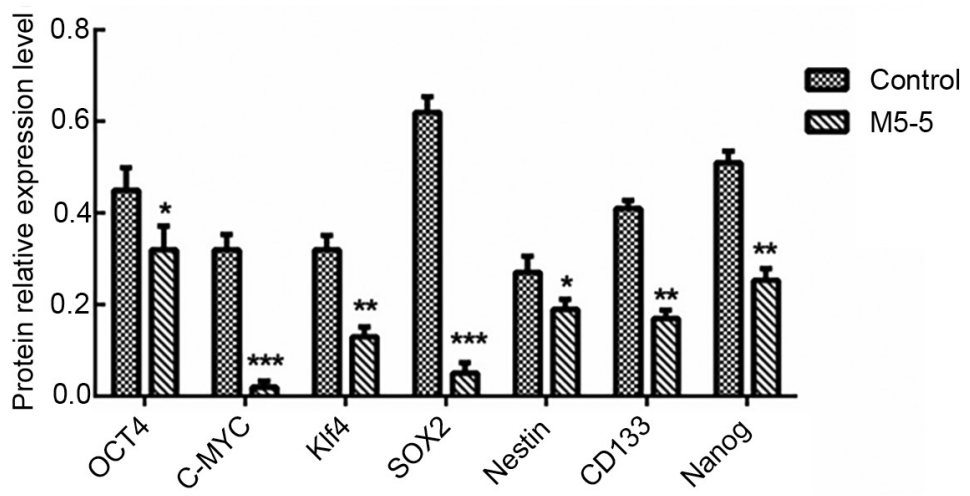

C

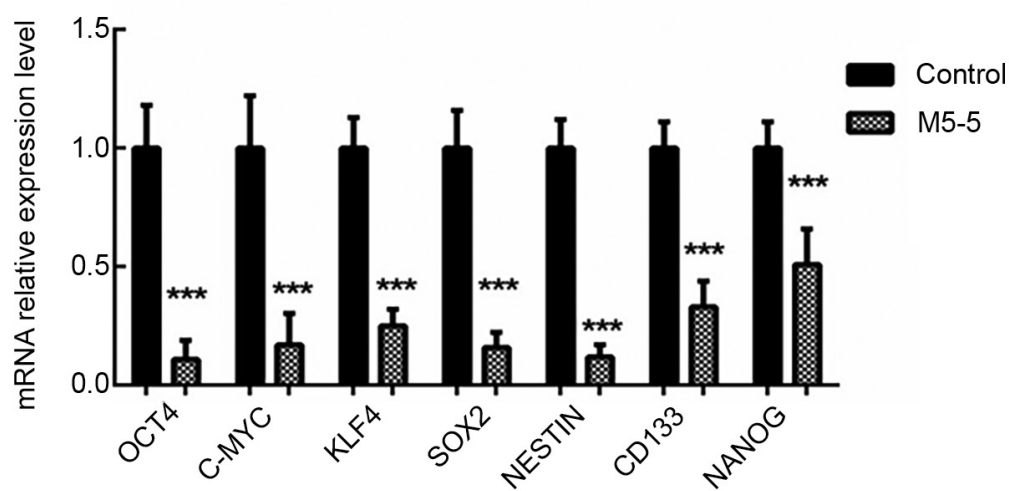

Figure 6. Detection of the effects of M5-5d intervention on GSC characteristics via western blotting and reverse transcription-quantitative PCR. (A and B) The protein expression levels of stem cell-associated genes, CD133 (130 kDa), c-Myc (57-70 kDa), Klf4 (65 kDa), SOX2 (35 kDa), Nestin (207 kDa), Nanog (42 kDa) and OCT4 $(45 \mathrm{kDa})$, were analyzed via western blotting. GAPDH (37 kDa) was used as an internal control. (C) Total RNA was extracted to quantify CD133, c-Myc, Klf4, SOX2, Nestin, Nanog and OCT4 at the mRNA level, which was normalized to GAPDH. ${ }^{*} \mathrm{P}<0.05,{ }^{* * *} \mathrm{P}<0.01$ and ${ }^{* * * *} \mathrm{P}<0.001$ vs. untreated GSCs as control group. Klf4, Krueppel-like factor 4; M, Mdivi-1; GSC, glioma stem cells.
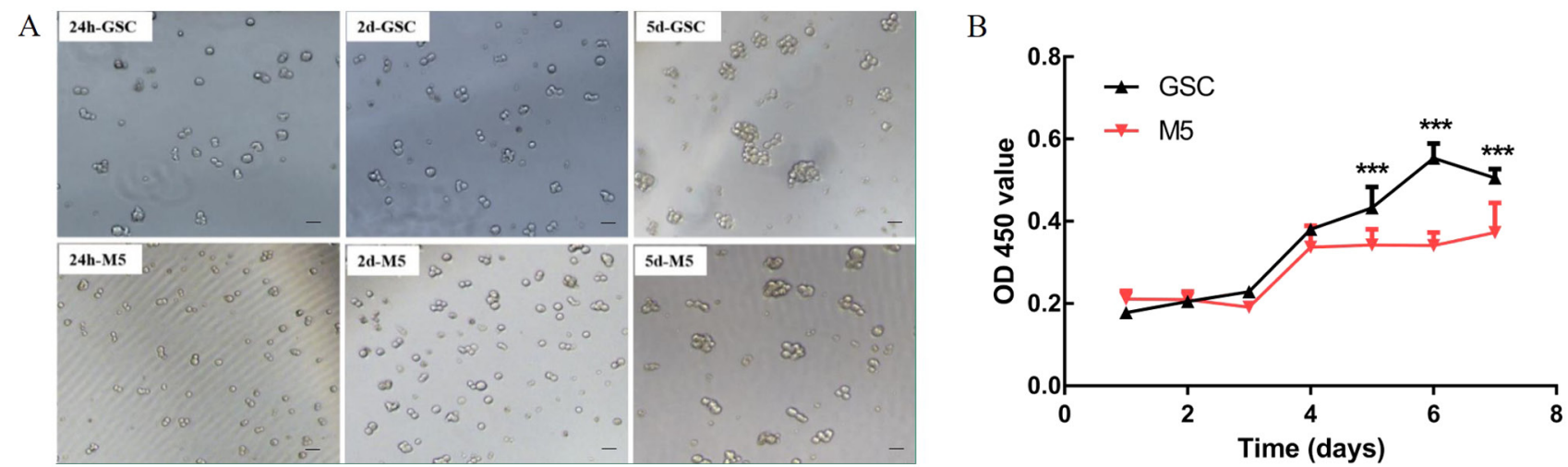

Figure 7. M5-5d intervention affects GSC proliferation. After 5 days of intervention with Mdivi-1 in GSCs, (A) the cells in good condition were selected and (B) proliferation was examined using Cell Counting Kit-8 assay. Scale bars, $50 \mu \mathrm{m} .{ }^{* * * *} \mathrm{P}<0.001$ vs. untreated GSCs at the same incubation time as control group. M, Mdivi-1; d, day; GSC, glioma stem cells; OD, optical density.

and the expression level of Bcl-2, which is an inhibitor of apoptosis, was decreased in the GSC group treated with $5 \mu \mathrm{M}$ Mdivi-1 for 5 days (all $\mathrm{P}<0.05$; Fig. 8). Therefore, it was revealed that GSC apoptosis was enhanced following treatment with Mdivi-1 for 5 days.

\section{Discussion}

It has been revealed that GSCs mediate the heterogeneity and drug resistance of glioma, and they are also associated with the prognosis of patients. Therefore, they may provide a novel direction for the treatment of glioma (40). The results of the present study revealed that GSCs could be induced to differentiate into neurons, oligodendrocytes and astrocytes. It was notable that in the process of GSC-induced differentiation, the results of GFAP identification demonstrated positive expression, but although the induced cells were $\mathrm{GFAP}^{+}$cells, it was difficult to determine whether they were astrocytes or glioma cells. It was difficult to distinguish the two cell types using morphology alone; however, the emergence of the 
A

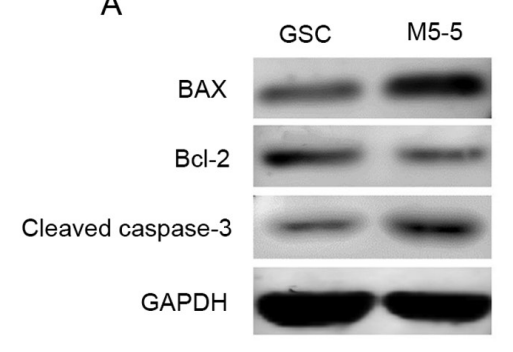

B

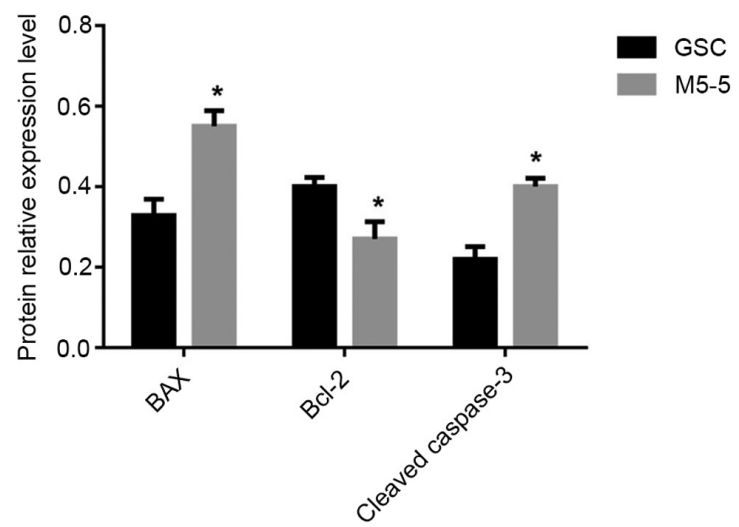

Figure 8. Expression levels of apoptosis-associated proteins in GSCs following M5-5d intervention. (A) Total proteins were isolated and the apoptosis-associated proteins Bax (20 kDa), Bcl-2 (26 kDa) and cleaved caspase-3 (17 kDa) were detected via western blotting. (B) The GSC only group was compared with the GSC group following M5-5d intervention. * $\mathrm{P}<0.05$ vs. untreated GSC as control group. M, Mdivi-1; GSC, glioma stem cells.

$\mathrm{GFAP}^{+}$cell population indicated that the cell model exhibited the potential for redifferentiation. Subsequently, the alterations in the expression level of stem cell-associated genes (CD133, C-MYC, Klf4, SOX2, Nestin, Nanog and OCT4) in the GSC population were determined by western blotting. These genes were expressed in GSCs. However, the expression level of these genes in GSCs was significantly decreased after the 5-day intervention with $5 \mu \mathrm{M}$ Mdivi-1, thereby altering the GSC characteristics. Subsequent experimental results revealed that $5 \mu \mathrm{M}$ Mdivi-1 significantly inhibited the proliferation of GSCs and altered the expression level of apoptosis-associated proteins, thereby potentially promoting apoptosis. At present, the specific surface markers of GSCs cannot be clearly determined (41). In order to establish a good therapeutic effect, GSCs must first be distinguished from normal stem cells or NSCs. Therefore, finding novel GSC-specific markers will aid follow-up research and clinical treatment.

It has been demonstrated that the proliferation and survival of tumor stem cells were closely associated with the function of mitochondria (42). The dynamic imbalance of the mitochondrial structure has been demonstrated to be closely associated with tumorigenesis (43). The present study established an innovative GSC model in vitro. A variety of methods were used to ensure the effective induction of GSCs. After the successful establishment of the cell model, the association between GSCs and mitochondria fission was investigated. Mdivi-1 can act as a protective agent in non-tumor cells and affect mitochondrial fusion/fission (33). Therefore, in the present study Mdivi-1 was selected to inhibit DRP1, thereby affecting the pathway of mitosis, and observe the role of mitochondria fission in GSCs. Mdivi-1 exhibits distinct effects in different cells (44), therefore it was necessary to examine the optimal intervention concentration and time of Mdivi-1. A number of different intervention durations and concentrations were investigated. The results indicated that the survival of cells treated with M5-5d was enhanced compared with those treated with M10-5d, which met the requirements of the cell status for subsequent experiments. In addition, the inhibition effect of M5-5d was most evident in terms of alterations in the DRP1 and Fis1 protein expression level among all concentrations and durations examined. Interestingly, DRP1 expression level was slightly decreased in M10-5d, but most importantly decreased in M5-5d. In terms of the survival status of the Mdivi-1 stimulated cells and the expression level of associated proteins, it was finally determined that stimulation with $5 \mu \mathrm{M}$ Mdivi-1 for 5 days was the most suitable intervention scheme. However, the present study only used Mdivi-1 as a DRP1 inhibitor to examine its function in GSCs and did not investigate how Mdivi-1 may interact with DRP1, which should be thoroughly examined in follow-up experiments.

Mitochondrial dynamics are considered to represent a novel perspective for understanding complex diseases, and the association between mitochondrial dynamics and diseases requires further investigation (45). In this case, mitochondrial targeting drugs are expected to interfere with tumor adaptability and promote the elimination of CSCs $(46,47)$. Although GSCs have gradually become a popular topic in clinical glioma research, there is still a number of problems that require further investigation. For example, an increasing number of studies $(48,49)$ have found that there is considerable plasticity between non-GSCs and GSCs subsets in glioblastoma, especially that non-GSCs differentiated during chemotherapy can transform into GSCs. However, how plasticity controls the mutual transformation of them remains unclear (50). At present, it is not clear what the actual molecular characteristics of GSCs are, and it is important to identify specific and reliable GSCs biomarkers (51). This requires constant investigation and improvement to provide theoretical and practical basis for basic research and clinical treatment. In conclusion, the results of the present study demonstrated that GSC cell models can be cultured in vitro by induction medium. Furthermore, after the specific inhibitor Mdivi-1 interferes with GSC, it can significantly reduce the expression of stem cell-related genes of GSC, inhibit the proliferation of GSC and promote the expression of apoptosis-related genes of GSC. Therefore, after using Mdivi-1 to affect the gene expression of DRP1 may represent a novel strategy of targeting glioma treatment.

\section{Acknowledgements}

Not applicable. 


\section{Funding}

The present study was funded by grants from the Ningxia Medical University School-level Scientific Research Project (grant no. XT2018013) and Ningxia High School First-class Disciplines (West China Top Class Discipline Project in Basic Medical Sciences, Ningxia Medical University; grant no. NXYLXK2017B07).

\section{Availability of data and materials}

The datasets used and/or analyzed during the present study are available from the corresponding author on reasonable request.

\section{Authors' contributions}

LZ and QH designed the study. LZ, HC, HT, JY and WG performed the research. $\mathrm{LZ}, \mathrm{HC}$ and $\mathrm{QH}$ analyzed the data. $\mathrm{LZ}, \mathrm{HC}$ and $\mathrm{QH}$ wrote and revised the manuscript. LZ and HC confirm the authenticity of the raw data. All authors read and approved the final manuscript.

\section{Ethics approval and consent to participate}

Not applicable.

\section{Patient consent for publication}

Not applicable.

\section{Competing interests}

The authors declare that they have no competing interests.

\section{References}

1. Moini J and Piran P (eds): Chapter 1 - Histophysiology. In: Functional and Clinical Neuroanatomy. Academic Press, pp1-49, 2020.

2. Singh SK, Hawkins C, Clarke ID, Squire JA, Bayani J, Hide T, Henkelman RM, Cusimano MD and Dirks PB: Identification of human brain tumour initiating cells. Nature 432: 396-401, 2004.

3. Pattabiraman DR and Weinberg RA: Tackling the cancer stem cells-what challenges do they pose? Nat Rev Drug Discov 13 497-512, 2014

4. Bao B, Ahmad A, Azmi AS, Ali S and Sarkar FH: Overview of cancer stem cells (CSCs) and mechanisms of their regulation: implications for cancer therapy. Curr Protoc Pharmacol; Chapter 14: Unit 14 25, 2013

5. Yan K, Wu Q, Yan DH, Lee CH, Rahim N, Tritschler I, DeVecchio J, Kalady MF, Hjelmeland AB, Rich JN, et al: Glioma cancer stem cells secrete Gremlin1 to promote their maintenance within the tumor hierarchy. Genes Dev 28: 1085-1100, 2014.

6. Sugiarto S, Persson AI, Munoz EG, Waldhuber M, Lamagna C, Andor N, Hanecker P, Ayers-Ringler J, Phillips J, Siu J, et al: Asymmetry-defective oligodendrocyte progenitors are glioma precursors. Cancer Cell 20: 328-340, 2011.

7. O'Brien CA, Kreso A, Ryan P, Hermans KG, Gibson L, Wang Y, Tsatsanis A, Gallinger S and Dick JE: ID1 and ID3 regulate the self-renewal capacity of human colon cancer-initiating cells through p21. Cancer Cell 21: 777-792, 2012.

8. Pece S, Tosoni D, Confalonieri S, Mazzarol G, Vecchi M Ronzoni S, Bernard L, Viale G, Pelicci PG and Di Fiore PP: Biological and molecular heterogeneity of breast cancers correlates with their cancer stem cell content. Cell 140: 62-73, 2010
9. Okano H: Stem cell biology of the central nervous system. J Neurosci Res 69: 698-707, 2002.

10. Zarco N, Norton E, Quinones-Hinojosa A and Guerrero-Cazares H: Overlapping migratory mechanisms between neural progenitor cells and brain tumor stem cells. Cell Mol Life Sci 76: 3553-3570, 2019.

11. Yu SC, Ping YF, Yi L, Zhou ZH, Chen JH, Yao XH, Gao L, Wang JM and Bian XW: Isolation and characterization of cancer stem cells from a human glioblastoma cell line U87. Cancer Lett 265: 124-134, 2008.

12. Wang R and Liu C: All-trans retinoic acid therapy induces asymmetric division of glioma stem cells from the U87MG cell line. Oncol Lett 18: 3646-3654, 2019.

13. Shahriyari L and Komarova NL: Symmetric vs. asymmetric stem cell divisions: An adaptation against cancer? PLoS One 8: e76195, 2013.

14. Lathia JD, Hitomi M, Gallagher J, Gadani SP, Adkins J, Vasanji A, Liu L, Eyler CE, Heddleston JM, Wu Q, et al: Distribution of CD133 reveals glioma stem cells self-renew through symmetric and asymmetric cell divisions. Cell Death Dis 2: e200, 2011.

15. Berika M, Elgayyar ME and El-Hashash AH: Asymmetric cell division of stem cells in the lung and other systems. Front Cell Dev Biol 2: 33, 2014

16. Najbauer J, Kraljik N and Nemeth P: Glioma stem cells: Markers, hallmarks and therapeutic targeting by metformin. Pathol Oncol Res 20: 789-797, 2014

17. Jin X, Jin X, Jung JE, Beck $\mathrm{S}$ and Kim H: Cell surface Nestin is a biomarker for glioma stem cells. Biochem Biophys Res Commun 433: 496-501, 2013

18. Zou Q, Yan Q, Zhong J, Wang K, Sun H, Yi X and Lai L: Direct conversion of human fibroblasts into neuronal restricted progenitors. J Biol Chem 289: 5250-5260, 2014

19. Wernig M, Tucker KL, Gornik V, Schneiders A, Buschwald R, Wiestler OD, Barde YA and Brüstle O: Tau EGFP embryonic stem cells: An efficient tool for neuronal lineage selection and transplantation. J Neurosci Res 69: 918-924, 2002.

20. Liu YJ, McIntyre RL, Janssens GE and Houtkooper RH Mitochondrial fission and fusion: A dynamic role in aging and potential target for age-related disease. Mech Ageing Dev 186: $111212,2020$.

21. Vyas S, Zaganjor E and Haigis MC: Mitochondria and cancer. Cell 166: 555-566, 2016

22. Taguchi N, Ishihara N, Jofuku A, Oka T and Mihara K: Mitotic phosphorylation of dynamin-related GTPase Drp1 participates in mitochondrial fission. J Biol Chem 282: 11521-11529, 2007.

23. Song M and Dorn GW II: Mitoconfusion: Noncanonical functioning of dynamism factors in static mitochondria of the heart. Cell Metab 21: 195-205, 2015.

24. Ryan JJ, Marsboom G, Fang YH, Toth PT, Morrow E, Luo N, Piao L, Hong Z, Ericson K, Zhang HJ, et al: PGC1 $\alpha$-mediated mitofusin-2 deficiency in female rats and humans with pulmonary arterial hypertension. Am J Respir Crit Care Med 187: 865-878, 2013

25. Senft $\mathrm{D}$ and Ronai ZA: Regulators of mitochondrial dynamics in cancer. Curr Opin Cell Biol 39: 43-52, 2016.

26. Seo BJ, Yoon SH and Do JT: Mitochondrial dynamics in stem cells and differentiation. Int J Mol Sci 19: 3893, 2018.

27. Kim D, Sankaramoorthy A and Roy S: Downregulation of Drp1 and Fis1 inhibits mitochondrial fission and prevents high glucose-induced apoptosis in retinal endothelial cells. Cells 9: $1662,2020$.

28. Ji WK, Hatch AL, Merrill RA, Strack S and Higgs HN: Actin filaments target the oligomeric maturation of the dynamin GTPase Drp1 to mitochondrial fission sites. Elife 4: e11553, 2015.

29. Yu R, Jin SB, Lendahl U, Nister M and Zhao J: Human Fis1 regulates mitochondrial dynamics through inhibition of the fusion machinery. EMBO J 38: e99748, 2019

30. Chen H, Detmer SA, Ewald AJ, Griffin EE, Fraser SE and Chan DC: Mitofusins Mfn1 and Mfn2 coordinately regulate mitochondrial fusion and are essential for embryonic development. J Cell Biol 160: 189-200, 2003.

31. Allegra A, Innao V, Allegra AG and Musolino C: Relationship between mitofusin 2 and cancer. Adv Protein Chem Struct Biol 116: 209-236, 2019.

32. Ruiz A, Alberdi E and Matute C: Mitochondrial division inhibitor 1 (mdivi-1) protects neurons against excitotoxicity through the modulation of mitochondrial function and intracellular $\mathrm{Ca}^{2+}$ signaling. Front Mol Neurosci 11: 3, 2018. 
33. Cassidy-Stone A, Chipuk JE, Ingerman E, Song C, Yoo C, Kuwana T, Kurth MJ, Shaw JT, Hinshaw JE, Green DR and Nunnari J: Chemical inhibition of the mitochondrial division dynamin reveals its role in Bax/Bak-dependent mitochondrial outer membrane permeabilization. Dev Cell 14: 193-204, 2008.

34. Valenti D, Rossi L, Marzulli D, Bellomo F, De Rasmo D, Signorile A and Vacca RA: Inhibition of Drp1-mediated mitochondrial fission improves mitochondrial dynamics and bioenergetics stimulating neurogenesis in hippocampal progenitor cells from a Down syndrome mouse model. Biochim Biophys Acta Mol Basis Dis 1863: 3117-3127, 2017.

35. Martinez-Outschoorn UE, Peiris-Pages M, Pestell RG, Sotgia F and Lisanti MP: Cancer metabolism: A therapeutic perspective. Nat Rev Clin Oncol 14: 113, 2017.

36. Livak KJ andSchmittgen TD: Analysis of relative gene expression data using real-time quantitative PCR and the 2(-Delta Delta C(T)) method. Methods 25: 402-408, 2001

37. Caren H, Stricker SH, Bulstrode H, Gagrica S, Johnstone E, Bartlett TE, Feber A, Wilson G, Teschendorff AE, Bertone P, et al Glioblastoma stem cells respond to differentiation cues but fail to undergo commitment and terminal cell-cycle arrest. Stem Cell Reports 5: 829-842, 2015.

38. Srikanth M, Kim J, Das S and Kessler JA: BMP signaling induces astrocytic differentiation of clinically derived oligodendroglioma propagating cells. Mol Cancer Res 12: 283-294, 2014.

39. Smith $G$ and Gallo G: To mdivi-1 or not to mdivi-1: Is that the question? Dev Neurobiol 77: 1260-1268, 2017.

40. Takashima Y, Kawaguchi A and Yamanaka R: Promising Prognosis Marker Candidates on the Status of EpithelialMesenchymal Transition and Glioma Stem Cells in Glioblastoma. Cells 8, 2019.

41. Kim WT and Ryu CJ: Cancer stem cell surface markers on normal stem cells. BMB Rep 50: 285-298, 2017.

42. Fiorillo M, Lamb R, Tanowitz HB, Cappello AR, Martinez-Outschoorn UE, Sotgia F and Lisanti MP: Bedaquiline, an FDA-approved antibiotic, inhibits mitochondrial function and potently blocks the proliferative expansion of stem-like cancer cells (CSCs). Aging (Albany NY) 8: 1593-1607, 2016.
43. Roberts ER and Thomas KJ: The role of mitochondria in the development and progression of lung cancer. Comput Struct Biotechnol J 6: e201303019, 2013.

44. Bordt EA, Clerc P, Roelofs BA, Saladino AJ, Tretter L, Adam-Vizi V, Cherok E, Khalil A, Yadava N, Ge SX, et al: The Putative Drp1 Inhibitor mdivi-1 is a reversible mitochondrial complex I inhibitor that modulates reactive oxygen species. Dev Cell 40: 583-594.e6, 2017.

45. Tilokani L, Nagashima S, Paupe V and Prudent J: Mitochondrial dynamics: Overview of molecular mechanisms. Essays Biochem 62: 341-360, 2018.

46. Semenza GL: Hypoxia-inducible factors: Coupling glucose metabolism and redox regulation with induction of the breast cancer stem cell phenotype. EMBO J 36: 252-259, 2017.

47. Peiris-Pages M, Martinez-Outschoorn UE, Pestell RG, Sotgia F and Lisanti MP: Cancer stem cell metabolism. Breast Cancer Res 18: 55, 2016.

48. Ohka F, Natsume A and Kondo Y: Chapter 15 - Clinical significance of epigenetic alterations in glioblastoma. In: Epigenetic Cancer Therapy. Gray SG (ed). Academic Press, Boston, pp339-350, 2015.

49. Garnier D, Renoult O, Alves-Guerra MC, Paris F and Pecqueur C: Glioblastoma stem-like cells, metabolic strategy to kill a challenging target. Front Oncol 9: 118, 2019.

50. Safa AR, Saadatzadeh MR, Cohen-Gadol AA, Pollok KE and Bijangi-Vishehsaraei K: Glioblastoma stem cells (GSCs) epigenetic plasticity and interconversion between differentiated non-GSCs and GSCs. Genes Dis 2: 152-163, 2015.

51. Yuan Y, Yan Z, Miao J, Cai R, Zhang M, Wang Y, Wang L, Dang W, Wang D, Xiang D, et al: Autofluorescence of NADH is a new biomarker for sorting and characterizing cancer stem cells in human glioma. Stem Cell Res Ther 10: 330, 2019.

(c) (7) $\ominus$ This work is licensed under a Creative Commons Attribution-NonCommercial-NoDerivatives 4.0 International (CC BY-NC-ND 4.0) License. 